Aus der Chirurgischen Abteilung des k. k. Krankenhauses Wieden in Wien.

\title{
Rückblick auf 2000 Operationen wegen Appendicitis.)
}

\author{
Von Prof. Dr. Julius Schnitzler.
}

M. H.! Meine Ausführungen an dieser Stelle sollen keine rein statistische Erläuterung der 2000 Operationen, welche ich bis zu Beginn dieses Jahres wegen Appendicitis ausgeführt habe, sein, und ebensowenig beabsichtige ich hier auf eine Besprechung der ganzen Appendicitisfrage einzugehen. Die angeführte Zahl der Operationen sowie die groBe Zahl von Appendicitisfällen, deren Verlauf ich ohne operatives Eingreifen verfolgt habe - in den letzten 7 Jahren an meiner Abteilung 242 Fälle mit 4 Todesfällen -, sollen nur die Grundlage zeigen, auf welche sich meine Ausführungen aufbauen, Ausführungen, die nur aphoristisch eine Reihe kontroverser Punkte aus dem so vielbearbeiteten Kapitel der Appendicitis erörtern sollen.

Wenn ich zunächst doch mit einigen Zahlen beginnen will, die ja das Gerippe dieses Berichtes bilden müssen, so sei erwähnt, daß von den 2000 Operationen 1063 sogenannte Intervalloperationen, 937 Operationen im akuten Stadium waren. Von den 1063 Intervalloperationen starben $5(0,47 \%)$, von den 937 im akuten Stadium Operierten $99(10,5 \%)$.

Die Differenz zwischen den beiden Geschlechtern in bezug auf ihr Befallenwerden von der Appendicitis ist so gering, daß man wohl von einer gleichmäBigen Beteiligung beider Geschlechter sprechen kann. Wenn ich aber hervorhebe, daB bei einer Form, der sogenannten chronisch anfallsfreien Form der Appendicitis, meine persönliche Zusammenstellung in Uebereinstimmung mit der letzten großen Berliner Sam melstatistik ein Ueberwiegen des weiblichen Geschlechtes, und zwar speziell junger Mädchen ergibt, so werde ich auf die Bedeutung dieser Tatsache bald zurückzukommen haben.

Als nicht mehr kontrovers betrachte ich die Frage, ob die Appendicitis tatsächlich in den letzten Jahren

1) Vorgetragen in der gemeinsamen Sitzung der luneren und Chirurgischen Sektion des XVl. Internationalen medizinischen Kongresses zu Budapest am 2. September 1909. (Siehe die Diskussion hierzu in No. 48, S. 2152.) 
zugenommen hat, oder ob diese Zunahme nur durch Aenderung der Diagnostik vorgetäuscht ist. Durch kritische Statistiken erscheint mir zweifellos festgestellt, daß nicht die Appendicitis, sondern nur ihre Diagnose häufiger geworden ist, und zwar, wie ich hinzufügen möchte, sowohl die richtige als die falsche Diagnose der Appendicitis. Erkannte man früher die - damals anders benannte - Appendicitis nur bei Vorhandensein des typisch lokalisierten Exsudates und war man förmlich blind für alle anderen uns heute geläufigen Formen und Stadien dieser Krankheit, so hat sich dann bei einer großen Anzahl von Aerzten und speziell von Chirurgen anstatt dieser Blindheit eine diagnostische Gesichtsfeldeinschränkung herausgebildet, die das Resultat hatte, daß man fast nur mehr die Appendicitis sah - allerdings zumeist mit Recht. Was aber die falschen Diagnosen, ich meine die Annahme einer Appendicitis dort, wo sie nicht vorliegt, betrifft, so erscheint es mir bemerkenswert, dab in vielen Fällen trotz der operativen Autopsie eine Korrektur der Fehldiagnose unterbleibt. Das gilt sowobl von akuten als von chronischen Fällen. Man hat sich so sehr daran gewöhnt, die eitrige Peritonitis als zumeist von einer Appendicitis ausgehend zu betrachten, daB mau nunmehr, wenn man bei einer Peritonitis keinen anderen Ausgangspunkt findet, die Appendix als den Vermittler betrachtet, auch wenn sie nur die peritonitischen Erscheinungen aufweist, die eben jedes intraperitoneale Gebilde unter diesen Umständen zeigt. Als Beispiel verweise ich nur auf einige Krankengeschichten Sonnenburgs in seinem für die heutige Sitzung erstatteten Referat. Bei eitriger Peritonitis und kaum veränderter Appendix wird diese als Ausgangspunkt der Peritonitis betrachtet. Wohl jeder Chirurg wird schon unter solchen Verhältnissen den Wurmfortsatz exstirpiert haben, ich habe dies in fünf derartigen Fällen getan, um mich erst nachträglich davon zu überzeugen, daß die nur an der Serosafläche schwerer erkrankte Appendix nicht der Ausgangspunkt der Erkrankung sein könne (drei dieser Fälle betrafen Kinder mit Pneumokokkenperitonitiden). Die Literatur zeigt nun, daB viele solche Fälle im Anschluß an die Operation genesen - auch meine Fälle wurden geheilt - , und vielfach wird daraus der Schluß gezogen, die Appendix müsse doch die Ursache der Erkrankung gewesen sein. Diese Schlubfolgerung ist durchaus nicht zwingend, und wenn Sonnenburg in seinem erwähnten Referat von Peritonitiden mit sekundärer Appendicitis spricht, so ist auch das noch ein zu weit gehendes Zugeständnis an die in solchen Fällen eben unberechtigte Diagnose einer Appendicitis. Und ebenso wie bei den erwähnten Fällen akuter Erkrankung, fällt es vielen Chirurgen schwer, wenn sie unter der Annahme einer chronischen Appendicitis einmal einen tatsächlich nicht kranken Wurmfortsatz entfernt haben, sich selbst zuzugestehen, daß reelle pathologisch-anatomische Veränderungen fehlen, und so mancher ist geneigt, aus einigen kleinen Hämorrhagien, aus einer Obliteration im peripherischen Teil der Appendix die Berechtigung zum Festhalten an der Diagnose der Appendicitis abzuleiten, worin ich auf Grund meiner persönlichen Erfahrungen diesen Chirurgen (und auch pathologischen Anatomen) nicht folgen kann. Auf diese Fragen werde ich an anderer Stelle näher eingehen, hier wollte ich nur andeuten, worin mir heute in der Diagnoseder Appendicitis zu weit gegangen zu werden scheint.

Aber diese Differenzen spielen eine verschwindende Rolle - für meine persönliche Statistik bei den akuten Fällen und chronischen Fällen zusammen kaum $2 \%$ - gegenüber der ungeheueren Häufigkeit der unzweifelhaften Appen. dicitis, und gerade diese Häufigkeit hat die verschiedensten Erklärungsversuche provoziert.

Unter den verschiedenen Richtungen, welche für die Auffassung über die Aetiologie der Appendicitis in Betracht kommen, bekenne ich mich als unbedingten Anhänger der mechanischen Auffassung. Nicht die Häufigkeit der Appendicitis erscheint erklärungsbedürftig, viel merkwürdiger erscheint es, daß der Wurmfortsatz, den man in Anlehnung an ein Wort von Reclus als Quadratur einer Sackgasse bezeichnen kann, so oft gesund bleibt. Meine Beobachtungen am Operationstisch haben mir den Abschluß resp. die Verengung der Appendix durch Narben, Schleimhautschwellungen,
Kotsteine und Torsionen - schon leichte Grade einer Torsion genügen, um hochgradige Abflußhindernisse zu bilden zu häufig gezeigt, um nach entlegeneren Erklärungen für das Zustandekommen und die deletären Steigerungen der Entzündungsprozesse zu suchen. Ich schließe mich jenen Autoren an, welche in erster Linie eine Enteritis in der Appendix als die Grundlage der weiterhin durch die speziellen mechanischen Momente oft so deletär verlaufenden Erkrankung annehmen. Bekanntlich ist ja in der letzten Zeit, insbesondere von Kretz, die hämatogene Entstehung der Appendicitis und speziell ihr Auftreten nach Angina in den Vordergrund gestellt worden, und man ist so weit gegangen, bei bestehender Enteritis die konsekutive Appendicitis durch Aufnahme von Bakterien aus dem enteritisch erkrankten Darm in die Blutbahn, also als hämatogen entstanden aufzufassen, eine ebenso gesuchte als unbegründete Auffassung. Daß Infektionskrankheiten und speziell Anginen manchmal der Appendicitis vorhergehen, kann ich bestätigen, und in ganz vereinzelten Fällen mag auch eine metastatische Entstehung nachweisbar sein, unter meinen Fällen ist sie mir allerdings nur in zwei Fällen nach dem klinischen Verlauf und dem Aussehen der Appendix wahrscheinlich. In allen anderen Fällen ist mir eine andere Art des Zusammenhanges plausibler. In einer Reihe von Fällen ist es gewiß die so oft die verschiedensten Infektionskrankheiten begleitende Enteritis, die in der früher angedeuteten Weise die Appendicitis einleitet. Mitunter liegen aber die Verhältnisse so, daß sich eine ganz andere Erklärung aufdrängt. Am besten wird dies an der Hand eines Falles klar.

Ich operierte einen Knaben, der im AnschluB an eine An gina an einer durch die typischen Symptome charakterisierten Appendicitis erkrankt war. Die Anamnese ergab, daß vor vielen Jahren die erste Attacke abgelaufen war und daß seither an jede Angina eine Appendicitis sich anschloß. Die Opera tion ergab einen fast völligen Verschluß der Appendix nahe dem Coecum und hinter dieser Stenose ein chronisches, frisch exazerbiertes Empyem.

Was Sprengel schon vermutet, daß „Zustandsänderungen" des Appendixträgers unter dem Einflub einer Angina (resp. anderen Infektionskrankheit) die Virulenz der in der Appendix längst bereit liegenden Bakterien erhöhen, das wird durch solche Beobachtungen wohl außer Zweifel gestellt.

In einem anderen Fall sah ich eine akute Appendicitis kurz nach dem Ablauf einer Diphtherie. Auch hier handelte es sich um ein frisch aufgeflackertes Empyem in einer fast ganz abgeschlossenen Appendix; die bakteriologische Untersuchung ergab im Appendixeiter nur Bacterium coli, im Rachen Diphtheriebazillen und Streptokokken.

Also auch hier keine hämatogene Infektion, sondern Dispositionsänderung.

Ganz abgesehen von der Kritik Aschoffs zu Kretz' Befunden erscheint es auch schwer verständlich, warum die mykotischen Embolien geradean der Appendix lokalisiert sein sollten. Dab solche Bakterienembolien auch am Ileum vorkommen, erwähnt Kretz gelegentlich. Unerklärlich blieb die Prädilektion der Appendix für embolische Erkrankung, während die Prädilektion für enterogene Erkrankung resp. deren Steigerung aus mechanischen Momenten naturgemäß sich ergibt. Auch muß doch hervorgehoben werden, daß wir bei Pyämie die Appendicitis nie zu sehen bekommen. Nicht uninteressant erscheint mir die Tatsache, daß sich unter meinen wegen akuter Appendicitis 0perierten eine grössere Zahl von Reisenden befindet, teils Fremden, die in Wien erkrankten, teils wieder Einheimische, die auf der Reise erkrankten und krank nach Wien zurückgebracht wurden. Die ungewohnte Kost erklärt wohl die Enteritis, die in der Appendix zur schwerwiegenden Erkrankung wurde.

Von den erkrankten Reisenden führt ein Uebergangsfall besonderer Art zu den traumatisch verursachten oder ausgelösten Appendicitiden.

Dieser Fall betraf einen Offizier, der im Vorjahr im Anschluß an den Distanzritt Budapest-Wien in den letzten Stunden dieses Rittes an einer schweren Appendicitis erkrankte, aus der ihn die Frühoperation befreite.

Daneben verfüge ich über zwei traumatisch ausgelöste Appendicitiden - eine nach Sprung von beträcht- 
licher Höhe, eine im Anschluß an einen Fußstoß gegen den Bauch.

In diesen beiden Fällen fanden sich Kotsteine, Gangrän und Perforation. Auch diese beiden Fälle wurden operativ geheilt.

Hier schließt sich naturgemäß die Erörterung über die Bedeutung des Kotsteins für die Aetiologie der Appendicitis an. Bekanntlich gestehen manche Autoren diesen Gebilden eine große Rolle bei der Entstehung der Appendicitis zu, während andere ihre Bedeutung leugnen, ja geradezu von einer Schutzwirkung des Kotsteines auf die von ihnen berührten Stellen der Appendixwand sprechen. Sprengel mibt ihnen mehr Bedeutung für die Entwicklung als für die Entstehung der Appendicitis zu. Hier kann doch wieder nurdie klinische Erfahrung entscheiden. Zunächst muß ich - mit Riedel und Sprengel - betonen, daß gerade bei den schweren, gangränösen Formen der Appendicitis die Kotsteine - und ich füge hinzu: grobe Kotsteine - besonders häufig gefunden werden. Vor allem aber muß ich betonen, $d a B$ bei den Intervalloperationen wirkliche Kotsteine äuBerst selten gefunden werd en. Ich betone "wirkliche Kotsteine", denn eingedickten Kot findet man oft genug bei den à froid-Operationen. Wenn man aber die typischen, solange für Kirschenkerne etc. gehaltenen Kotsteine gerade bei den schweren akuten Anfällen findet, so kann man nur zwei Möglichkeiten annehmen: entweder, dab unter dem Einflub des akuten Anfalles diese Gebilde sich rasch formieren - und das ist doch recht unwahrscheinlich - oder, und dies ist eben das Wahrscheinliche, dab bei Vorhandensein echter Kotsteine ein akuter, schwerer Anfall nicht lange auf sich warten läßt. Man hat ja bekanntlich diese Kotsteine und ihre Konsequenzen schon vielfach mit den Gallensteinen und ihren Schicksalen verglichen. Der Vergleich läßt sich noch viel besser durchführen, wenn man nicht an das Verhalten der Gallensteine in der Gallenblase und im Ductus cysticus, sondern an das Verhalten des Darmes und der Gallensteine beim Durchbruch großer Gallensteine in den Darm und die Erscheinungen beim Gallensteinileus denkt. Ich will dies hier nicht weiter ausführen.

In Hinblick auf die sonderbare Auffassung von der Schutzwirkung der Kotsteine auf die Appendixwand will ich nur darauf hinweisen, dab zirkuläre Dekubitusgeschwüre oder wenigstens deutliche Druckmarken in der Schleimhaut der Appendix ein typischer Befund sind, daß ich mehrmals bei Frühoperationen den an diesen Befunden schuldtragenden, schließlich ausgetriebenen Kotstein im Coecum fühlen konnte, daß ferner bei zahllosen à froid-Operationen zirkuläre Narben im Bereiche der Appendix als Endresultat der erwähnten Dekubitusgeschwüre gefunden werden, lauter Befunde, die von der "Schutzwirkung" des Kotsteins auf die Appendixwand wenig verraten!

Die einst als so bedeutungsvoll betrachtete Rolle der Fremdkörper für die Appendicitis ist wohl ausgespielt - allerdings nur im Wurmfortsatz; in den Köpfen der Laien und auch noch mancher Aerzte lebt diese Idee noch immer. Je einmal fand ich einen Holzspahn und ein Kümmelkorn in der Appendix, das war die ganze Fremdkörperausbeute in 2000 Fällen!

Gestatten Sie mir nun einige Bemerkungen über die Bakteriologie der Appendicitis in ihren Beziehungen zur Klinik. Haim hat die im Einzelfall nachgewiesene Bakterienart zum pathologisch-anatomischen Bild und zur Prognose der Appendicitis in bestimmte Beziehungen zu bringen versucht. Er betonte die Malignität der Streptokokkenappendicitis, als deren typischen Ausdruck er die ganz kleinen Perforationen ohne Kotstein ansah, und hob demgegenüber die relative Gutartigkeit der Koli-Appendicitis hervor. Leider habe ich nichts davon bei meinen Untersuchungen bestätigt gefunden. Weder die Prognose auf Grund des bakteriologischen Befundes, noch die Entstehung der kleinen Perforationen gerade durch Streptokokken kann ich als gesetzmäßig gelten lassen. Ausschlaggebend ist ja nicht die Art, sondern die Virulenz der Bakterien, und es fragt sich, ob wir für diese ein Kriterium haben. Zweifellos besitzen wir ein solches. Es ist das klinische Bild, das der Patient darbietet.

Als die Frühoperation aufkam, als man sich so oft über- zeugen konnte, daß trotz eines durchaus nicht schweren klinischen Bildes ein sehr schwerer pathologisch-anatomischer $\mathrm{Be}$ fund an der Appendix bestehen könne, hat man diese Diskrepanz mit Recht als Motiv für die Frühoperation herangezogen. Ich habe schon auf dem Chirurgen-Kongreb 1905 meiner Ansicht dahin Ausdruck gegeben, daß die Prognose doch in erster Linie dem klinischen Bild und nicht dem pathologischanatomischen Befund entspricht, d. h. daß, wenn wir bei gutem klinischen Befinden operieren, die Prognose auch bei sehr bösem pathologisch-anatomischen Befund gut ist. $\mathrm{Zu}$ den Kriterien des guten Befindens gehört allerdings außer entsprechender Herztätigkeit und normalem Sensorium erhaltene Darmtätigkeit und Fehlen des Meteorismus. Operieren wir in diesem Stadium, d. h. bei klinisch-symptomatologisch günstigem Befinden, und verschlechtert sich nach der Operation das Befinden des Patienten, so dürfen wir nach meiner Ansicht diese Verschlechterung nicht als einfach selbstverständliche Folge des schweren pathologischanatomischen Befundes auffassen - sonst hätte sich ja der Kranke schon vor der Operation schlecht befinden müssen sondern wir müssen dann mit der Tatsache rechnen, daß die Operation die Verschlechterung des Zustandes nach sich geführt hat. Allerdings zeigt uns die schwere pathologisch-anatomische Veränderung der Appendix, daß eine Gefahr dem Kranken wohl bevorstand, daß also ein Eingriff zur Beseitigung dieser Gefahr berechtigt war. Nun, wer über die nötige Selbstkritik verfügt, um sich in dieser Beziehung keinen Selbsttäuschungen hinzugeben, wird zu einer richtigen Indikationsstellung bezüglich der Appendicitisoperation gelangen. (Broca hat in recht anschaulicher Weise die Psychologie der Operateure in bezug auf diesen Punkt geschildert.)

Wenn ich gesagt habe, daß die Prognose des appendizitischen Anfalles von der Virulenz der Bakterien abhängt, so muß ich nun noch einen zweiten ausschlaggebenden Faktor anführen: die Lagerung der Appendix. In einer Reihe von Fällen ist der Wurmfortsatz - entweder durch Lagerung hinter dem Coecum und Colon ascendens oder hinter dem Mesenterium ileocoecale - im praktischen Sinne extraperitoneal gelagert. DaB dadurch die Gefahr der Entstehung einer allgemeinen Peritonitis wesentlich eingeschränkt wird, ist selbst verständlich. Hervorheben mub ich aber, daB es sich bei dieser Lagerung um angeborene Verhältnisse handelt. Einen in analoger Weise wirkenden Schutz infolge von Verwachsungen, die sich währendeiner appendizitischen Attacke ausgebildet haben und nun für die späteren Anfälle förmlich als Schutzorgane wirken, sieht man kaum je. Jedenfalls ist ein solches Vorkommnis praktisch nicht in Betracht zu zieherl, und die daraufhin von so vielen Seiten aufgestellte Behauptung, dab nach einem schweren Anfall die weiteren Anfälle eben infolge der schützenden Verwachsungen weniger gefährlich seien, ist durchaus falsch.

Nur wenige Bemerkungen möchte ich an dieser Stelle über die von verschiedenen Seiten besprochene klinische Diagn os e der Lagerung der Appendix einschalten. Was an dieser Diagnostik selbstverständlich ist: daß ein nach aufwärts gelagerter Wurmfortsatz Schmerzen in der Gallenblasengegend, eine im Douglas gelegene Appendix bei einer Entzündung Blasensymptome hervorrufen kann, findet sich in der Regel bestätigt. Für die ganz regellose und im Einzelfalle überraschende Lokalisation der Schmerzen haben wir aber keine Erklärung.

So sah ich eine akute Appendicitis (Operation am zweiten Tag), die mit den heftigsten Schmerzen in der linken Unterbauch. gegend begonnen hatte. Die Operation zeigte den vom Colon ascendens gedeckten Wurmfortsatz steil nach aufwärts bis nahe an die untere Leberfläche verlaufend.

Solche Inkongruenzen findet man, soweit meine Aufzeichnungen reichen, in etwa $10 \%$ aller Fälle.

Erlauben Sie mir, meine Herren, nun einige Worte zur Diagnostik und Fehldiagnostik der Appendicitis. Die typischen und wichtigen Symptome, der lokale Schmerz, die Muskelspannung, das Erbrechen, später die Symptome der fortschreitenden und allgemeinen Peritonitis brauchen ja nicht erst erwähnt zu werden; dieser Teil der Symptomatologie beginnt glücklicherweise Allgemeingut der Aerzte zu werden. 
Ebensowenig soll hier die häufige Verwechslung mit Adnexerkrankungen, Extrauteringravidität, Cholelithiasis, Nephrolithiasis etc. erwähnt werden. Daß beginnende Pneumonie mil appendizitischen resp. rechtseitigen peritonitischen Érscheinungell einhergehen kann, ist gerade in der letzten Zeit mehriach betont worden.

Ich selbst war dreimal in der Lage, Operationen, zu deren dringlicher Auslunrung ich gerufen worden war, unter f'eststellung einer Pneumonie abzulehnen. Die Art der Atmung, die F'eststellung, dabs bei geduldiger Prüfung die Bauchdeckenspannung überwindbar war und dann ein sehr tieter, allmählicher Druck ohne besondere schmerzen ertragen wurde, und schlieblich das ganze, nicnt immer in Worten determinierbare klinische Bld scnutzten mich vor der Fehidiagnose.

Wenig gekannt ist die Tatsache, daß im Anschlub an Klismen resp. hohe lrrigationen eln Krankheitsbild vorkommen kann, das einer Peritonitis resp. Appendicıtis täuschend gleicht. Schmerz, Erbrechen, Bauchdeckenspannung, sogar vertallenes Aussehen vereinigen sich, um die Fehldlagnose zu provozierell, und ich habe elnmal in einem derartigen Fall gemeinsam mit dem sehr erfahrenen und besonnenen Hausarzt auch die operative Konsequenz aus dieser Fehldiagllose gezogen. Die Patıentin, die in spàter Nachtstunde operielt wurde, wal am nächsten Morgen ganz gesund und sehr dankerfüllt. Die Érklärung für diese diagnostısche l'äuschung - ich habe spater in der englischen Literatur analoge Falle angeführt getuıden - ist wohl in del' durch eıne plötzliche starke Fullung von Loecum ev. auch Appendix ausgelöstell Reizung zu suchen.

Eis ist weder möglich, noch wäre es angezeigt, an dieser Stelle auf alle Mögllchkeiten einer Verwechslung zwischen akuter Appendicitis und anderen Erkrankungen einzugehen. Nul elne allgemeine Regel möchte ich aufstellen. F'sndet man bel der Uperation, die man wegen tirscheinungen allgeineller Peritonitis ausgetuhrt hat, keine Peritonitis und dabel aucli die nach der Entfernung natürlich auch in aufgescinıttenein Lustande untersuchte Appendix nicht wirkuch krallk, so suche nlall nicht welter in der Bauchhöhle herum. Das F'ehlen der Pelitonitis macht diese Unterlassung zur Pflicht. Findet mall abel bel der Laparotomie Peritonits, so begnüge man sich nicht init der Feststellung eines $\mathrm{zweltelhaften} B \mathrm{~B}$ fundes an der entfernten Appendix. 'Leigt diese an der schleim haut nicht ınakloskopısch elndeutıge Veränderungen, so wird die Ul'sache der Peritonitıs eben anderwärts zu suchen und zu beseltigell sein, und der Ertahrene - die anteoperatıve F'ehldıagnose bleibt auch diesem nicht immer erspart wird oft gellug aus del Beschafienheit des peritonealen Exsudates daruber orientiert seln, ob er den Erkrankungsherd in den Adıexen, ellein Ulcus ventriculi, der Gallenblase etc. zu suchen liat. Melir als diese Andeutungen kann ich hier nicht geben.

Auch alle Möglichkeiten der Fehldiagnose bei der chronisclı $\theta$ n Appendicitis konnen hier nicht besprochen werden. Nur die tür die lilulkationsstellung der Intervalloperation wichtıgeren Punkte sollen erwähnt werden. Ist ein vorausgegangener Anfall akuter Appendicıtis in der Anamnese sichergestellt, so genügt dıes aus später zu erörternden Giründen für die Indlkatiolisstellung vollkominen. s'chwieriger steht die Frage bezuigilch der sogenannten chronisch antallstreien Appendicitis. Was zunächst die pathologisch-anatomische Seite dieser F'rage betritıt, so schliebe ich mich jenen Autoren (A s ch off u. a.) an, welche elne primär chronısche Appendicitis nicht anerkennen. E's handelt sich aber um die klinisch-praktische Seite der Frage: Kann der Wurmfortsatz krank sein, ohne dab jemals Symptome eines akuten An. falles bestanden haben? Diese Frage muß unbedingt bejaht werden. Ich habe schon früher auf die schleichende Entstehung stenosierender Narben in der Appendix infolge von Dekubitus durch Kotsteine hingewiesen, hinter denen sich bald ein Hydrops, bald ein Empyem entwickelt. Darüber, dab solche Appendices eine Gefahr bedeuten und entfernt werden müssen, sind wohl alle einig, und dab dauernde Beschwerden - Koliken, Druckgefühl etc. - hier ihren Ursprung haben können, ist plausibel. Daß solche Schmerzen falsch (im Sinne des Arztes!) lokalisiert werden und daß wir dann von einer larvierten, schwer erkennbaren Appendicitis spreclien dürfen, ist wohl bekannt. Die Schwlerigkelt für die Indıkationsstellung liegt aber darin, daß all diese positiven und negativen Kriterien und dazu noch die Druckemptindlichkeit in der lleozökalgegend auch bei Leuten vorkommt, deren Appendix zweifellos normal ist. Unter diesen Umständen sind Irrtümer in der Diagnose und Indikationsstellung der als chronisch anfallstrei diagnostizierten Appelldicitis unvermeidlich, und während dıe Chirurgen in den symptomlos entstehenden Stenosen und Ëmpyemen und deren Gefahren eine Kechtfertigung für ihre möglıchst radikale Indlkationsstellung finden, gibt es eine Reihe von Internisten, die durch den Anblick einer exstirpierten, gesunden Appendix zu Bekämpfern der à froid-Operation geworden sind. Irıtümer sind, das ist ja durch die eben gemachten Ausführungen motiviert, unvermeldllch. Jali die Entfernung einer gesunden Appendix im Verglelch zur Belassung eines schwer kranken, wenu auch zunäcnst fast symptomlosen Wurmfortsatzes kaum eln Unglück darstellt, ist schon oft und mit Recht betont worden. Aber darum soll dle Gesundhelt einer ırrtumlıch entferıten Appelldix - diese Gesundheit mub sich auf Wandbeschufiellheit, tiestalt, Beweglichkelt etc. beziehen aucll rucknallos zugestanden und die Berechtigung zur Operation in den allgemein praktischen Gesichts punkten und licilt in ein par bedeutuligslosen schlolmhautblutullgen gesucht werden! Es ist dann statt einer kurativen elle prophylaktısche Appendektomie gemacht worden, und ich kann dalin durchaus keln Unglück sehen. Allerdıngs muls aber hıer ell Punkt besonders erörtert werden. Ist elle solche an den Schmerzen nicht schuldtragende Appendix - und ich glaube an die Auslösung von sichmerzen von einer inakroskopiscll unveränderten, normal gelagerten Appendix aus nicht - entferut worden, so entsteht llaturgemàll die F'rage, wo die Ursache der sichmerzen liegt. Ist elle tullerocolitls die Ursaclle der Beschwerden, worauf besonders Dieulatoy, bekanntlich ein äuberst operatlonsireundlicher Forschel, hligewiesell hat, so wird wenigstens die diatetiscn-physikallsche 'l'1lerapıe nun ohne Heminungen erschöpft werden konilen. Bekannt sind die hier nur anzudeuteliden Unannehmlıchkeıten, wenn hinter der scheinbaren Appendıcitıs elle Cholelithiasis, eine Nephrolithlasis oder ein Ulcus ventrıcull versteckt war. Siehr haufig handelt es sich abor um psychlsch-neurousche Vorgange, und die im Anfung meiner Austührungen betonte hönere Hauflykelt der sogenannten chronisch allablstielen Appendicitis belm weiblichen Geschlecht in der Beruner salnmelstatıstlk ulı in ineiner personlıchen Statistık ist wohl recht charakteristisch. Die tintfernung elner llormalell Appendix bei elner nervosen Patientin, ell Vorkommnis, dessell nicht vereinzeltes Volkommen kein ehrlicher Chirurg leugnen wırd, ist fül den Operateur gewlb mit kelnem Hocngefuhl verbunden, für die Patientin aber doch zumeist von Vortell und kaum je eine Gefalır. Aber ich kann nicht unterlassen, auf elle Fehlerquelle bei der Diagnose der chronisch antallsfreien Appendicitls hinzuweisen, nannich auf die begınnende resp. latente Ĺ ungentuberkulose. Es ist ja längst bekannt, dabj beginnende Lungentuberkulose oft mit Magenbeschwerden und auch init Magenschmerzen einhergeht. Heute wird doch jeder solche Fall auf Lruckempfindlichkeit in der Blınddarıngegend untersucht, diese Empfindlichkeit oft genug gefunden, und der Weg zur Fehldiagnose ist eingeschlagen!

Ich wurde auf diesen Zusammenhang durch einen Fall aufmerksam, den ich vor etwa sechs Jahren operiert habe. Typische chronische Beschwerden; die schon von Internisten behandelte Patientin wird zur Appendektomie in das Spital geschickt und nach kurzer Beobachtung operiert. Keine wesentlichen Veränderungen am Wurmfortsatz. Wundheilung normal. Vier Wochen nach der Operation eine innerhalb weniger Minuten letal verlaufende Haemoptoë! Die Sektion ergab einen kleinen, klinisch kaum nachweisbaren tuberkulösen Herd in der rechten Lungenspitze. Der Darm frei von tuberkulösen Veränderungen.

Ich habe seit der Beobachtung dieses Falles begreiflicherweise dieser Irrtumsquelle besondere Aufmerksamkeit geschenkt und beispielsweise einen Fall von angeblicher chronischer Appendicitis zu operieren abgelehnt, weil ich eine beginnende Lungentuberkulose als hochwahrscheinlich annahm. Der 
Patient wurde anderwärts appendektomiert. Im Anschlub an die Operation rapides Fortschreiten der Lungentuberkulose und Tod nach wenigen Monaten. Ich habe mich ferner durch Umfrage bei mehreren erfahrenen Internisten darüber vergewissern können, daß eine solche diagnostische, in diesen Fällen oft folgenschwere Verwechslung, wenn auch recht selten, vorkommt. Gerade für die Klärung derartiger Fälle hat sich mir in letzter Zeit die 0 phthalmodiagnostik sehr gut bewährt. (Von den Verwechslungen zwischen gewöhnlicher Appendicitis und Ileozökaltuberkulose ist natürlich hier nicht dic Rede.)

Nach diesen, natürlich nur aphoristischen Bemerkungen über die Diagnostik will ich auf Grund meiner Resultate die Indikationsstellung und Therapie besprechen. Unter meinen 2000 Operationen sind neben 1063 Intervalloperationen 937 im akuten Stadium ausgeführte Eingriffe. Nur letztere sollen zunächst weiter besprochen werden. Von den 9370 perierten waren 112 AbszeBinzisionen (darunter 23 radikale und 2 perineale bei Douglasabszessen) und 825 radikale Eingriffe. Von letzteren waren 444 sogenannte Frühoperationen und 381 später als 48 Stunden nach Beginn der Erkrankung ausgeführt. Ich schalte hier ein, daß die Termini Frühoperation und Intervalloperation meiner Ansicht nach ausschlieflich im klinischen Sinne gebraucht werden dürfen. Es geht nicht an, wenn bei einer Intervalloperation eingedickte Eiterreste gefunden werden, diese Operationen - besonders, wenn sie schlecht ausgehen - zu den während des Anfalles unternommenen zu zählen, und ebensowenig darf man, wenn der wegen akuter Erscheinungen Operierte noch am Tage vor der Operation ohne alle Krankheitserscheinungen seiner gewöhnlichen Beschäftigung nachgegangen ist, wenn nun bei der Operation Gangrän oder eine Perforation der Appendix zu finden sind, diesen Fall von der Liste der Frühoperationen streichen. Wir dürfen diese aus rein praktischen Gesichtspunkten geschaffenen Termini der Früh. und Intervall-Operation nur vom klinischen Standpunkt aus betrachten; benützen wir sie vom pathologisch. anatomischen Standpunkt aus, so gelangen wir zu Selbstverständlichkeiten, die keinen Wert für unsere therapeutischen Entschließungen haben. Unter meinen Frühoperationen habe ich 29 , d. s. $6,5 \%$ Mortalität, und zwar unter den am ersten Tag operierten 153 Fällen $7=4,6 \%$, unter den am zweiten Tag operierten 29122 Todesfälle. Besonders die Mortalität am ersten Tag ist größer, als man erwarten sollte. Aber gerade unter diesen Todesfällen befindet sich eine Embolie der Pulmonalarterien, eine Pylephlebitis und eine schwere Bauchdeckenphlegmone bei einem Diabetiker, während nur vier von diesen am ersten Krankheitstag operierten 153 Patienten einer Peritonitis erlagen. An anderer Stelle werde ich auf die Kritik der Todesfälle nach Frühoperationen näher eingehen. Hier sei nur erwähnt, daß es sich stets um destruktive Appendicitiden handelte.

Wenn nun $4 \pm 4$ Frühoperationen 381 spätere radikale Operationen und 112 AbszeBinzisionen (also 493 Spätoperationen) gegenüberstehen, so zeigt dies im Vergleich mit vielen anderen Statistiken, bei denen die Frühoperationen an Zahl viel weiter hinter den an späteren Krankheitstagen unternommenen Operationen zurückbleiben, daß ich offenbar nicht alle in späteren Tagen der Erkrankung eingelieferte Patienten operierte. Tatsächlich sind allein in den letzten sieben Jahren an meiner Abteilung 242 Appendicitiden unoperiert geblieben und von diesen nur vier gestorben. Drei von diesen Todesfällen betrafen moribund überbrachte Kranke, die nach ganz kurzer Zeit starben; der letzte Fall wurde in noch operationsfähigem Zustand überbracht, verweigerte aber die Vornahme der Operation - ein heute wohl seltenes Vorkommnis. Eine e infache Gegenüber stellung der 242 nicht operierten Fälle mit nur vier Todesfällen und der 937 Fälle mit 10,5\% Mortalität würde natürlich ein ganz falsches Bild der Resultate interner und chirurgischer Behandlung geben. Vor allem befindet sich unter den nicht operierten Fällen eine ganze Reihe äuferst leichter Erkrankungen, deren Operation, da nach wenigen Stunden alle Erscheinungen schwanden, nicht in Frage kam. Anderseits wurde ja bei allen Kranken, bei denen im Moment der Aufnahme in das Spital entweder infolge fehlender Zustimmung oder wegen Stillstand resp. Rückgang der Erkrankungen zunächst nicht operiert wurde, stets bei Verschlimmerung des Krankheitsbildes operativ eingegriffen, sodal also für die Mortalität der nicht operierten Fälle kein Material übrig blieb. Allerdings mub ich hervorheben, daf gerade die Todesfälle bei den im akuten Stadium Operierten nur in einem einzigen Fall einen zunächst abwartend und erst bei Zunahme der schweren Erscheinungen Operierten betrafen. Sonst betrafen die Todesfälle durchwegs solche Patienten, welche wegen des schweren Krankheitsbildes sofort operiert wurden. Und ich muf hier auf die gangbare Behauptung eingehen, dab man aus den klinischen Symptomen kein Urteil über die Schwere des Krankheitsfalles fällen kann.

Es ist richtig, daß man oft genug den pathologisch-anatomischen Zustand der Appendix vor der Operation falsch beurteilt, obwohl wir in dieser Beziehung in den abgelaufenen 15 Jahren unendlich viel gelernt haben, und die These, dab man bei Eröffnung der Bauchhöhle oft durch die unerwartete SchwerederVeränderungen überraschtwird, is theute schon deplaciert oder ein Zeichen unzureichender Er. fahrung. Vor allem haben wir die einzelnen Symptome richtig zu bewerten gelernt, und darum wird der erfahrene Chirurg sich über die Schwere der Erkrankung kaum mehr täuschen, und er wird zumeist sogar die Qualität der pathologisch-anatomischen Erscheinungen, d. h. ob ein destruktiver Prozeb vorliegt oder nicht, richtig beurteilen. Ich habe aber oben schon gesagt, dab nicht die Schwere der pathologisch-anatomischen Veränderungen der Appendix die Gefahr der Krankheit allein bestimmt, und darum erlaubt uns auch die wesentlich verfeinerte Diagnostik nicht, am ersten oder zweiten Tag der Erkrankung zu entscheiden, in welche Gefahren. klasse - um mich eines versicherungstechnischen Ausdrucks zu bedienen - der einzelne Fall gehört. Daher die Berechtigung der Frühoperation, die bessere Resultate ergibt als die Gesamtheit der unoperierten Appendicitiden. Ich kann aber nicht zugestehen, daB diese Unsicherheit in der Prognose während der ganzen Dauer einer akuten Appendicitis anhält und daher die Indikationsstellung in jedem Moment der Erkrankung unverändert bleibt. Jeder auf diesem Gebiete erfahrene Arzt mul zugestehen, dab in einer ganzen Reihe von Fällen der volle Rückgang aller schweren Symptome und die Bildung eines scharf abgegrenzten Exsudates es uns ermöglichen, für diesen Fall eine beinahe absolut günstige Prognose zu stellen, und dab in diesen Fällen ein aufmerksamer und geschulter Beobachter, wenn doch noch ein neuerliches Weiterschreiten der Krankheit stattfindet, die warnenden Symptome rechtzeitig wahrnehmen kann. Nur wenn auch in dem Stadium der vollen Abgrenzung des Tumors, also kaum je früher als vom dritten Tag an, die Operation, und zwar die radikale Operation unter Mitentfernung der Appendix, befriedigendere Resultate geben würde als die von so vielen erfahrenen Chirurgen und auch von mir empfohlene individualisierende Behandlung, könnte ich dem Prinzip des je derzeitigen, wahllosen Operierens bei der akuten Appendicitis zustimmen. Ganz abgesehen davon, daß fast alle Statistiken bei den radikalen Operationen nach dem dritten Tage mit Mortalitätsziffern um $20 \%$ und höher belastet sind, ergibt die vorurteilslose Betrachtung von Einzelfällen, daß die wahllose Operation in allen Stadien durchaus nicht immer von Vorteil ist, vielmehr zweifellos oft Schaden stiften kann. Nicht theoretische Bedenken haben mich zum Anhänger der individualisierenden Richtulng gemacht, sondern traurige, aber lehrreiche Erfahrungen, die teils meiner eigenen operativen Tätigkeit aus viel früherer Leit entstammen, teils aber Beobachtungen an von Fachkollegen operierten Patienten betreffen.

So habe ich vor einer längeren Reihe von Jahren kurz hintereinander zwei ganz analoge Fälle zu beklagen gehabt, die folgendermaßen verliefen: Die Patienten, die sich am vierten resp. sechsten Tag der Erkrankung befanden, wiesen den typischen zirkumskripten, dabei nicht sehr schmerzhaften Tumor in der Ileozökalgegend auf. Die Anamnese ergab voraufgegangene peritonitische Symptome, die seit den letzten zwei- bis dreimal 24 Stunden geschwunden waren. In beiden Fällen schloß sich an die unter allen Kautelen - Abgrenzung des Operationsgebietes durch Gaze, entsprechende Drainage etc. - ausgeführte radikale Operation, wobei 
jedesmal eine perforierte Appendix entfernt wurde, eine rapid verlaufende Peritonitis an, die zweifellos durch die Operation ausgelöst war. Und vor wenigen Jahren sah ich ein zehnjähriges Kind, dessen Operation ich mit Rücksicht auf den ganzen Befund - zirkumskripter Tumor am fünften Tage der Erkrankung, keinerlei peritonische Erscheinungen - als derzeit unnötig und nicht ungefährlich abgelehnt hatte, nach der trotz meines Abratens von einem Fachkollegen ausgeführten radikalen Operation an einer an die Operation sich anschließenden Peritonitis zugrunde gehen.

Solche Fälle blieben gegenüber gewib recht zahlreichen auch in diesem Stadium mit gutem Erfolge ausgeführten Operationen für mich entscheidend. Im Stadium des scharf um. schriebenen - natürlich nicht durch Fluktuation als großen AbszeB agnoszierbaren - Tumors ohne alle peritonitischen Symptome rate ich unbedingt zunächst zum Zuwarten.

Nur eine Voraussetzung ist an die individualisierende $\mathrm{Be}$ handlung geknüpft: der Kranke muß in einer Anstalt unter entsprechend häufiger Kontrolle eines hinreichend erfahrenen und gewissenhaften Arztes stehen! Wenn man aber durchsetzen kann, daß jeder Patient sich in jedem Stadium der Appendicitis operieren läßt, dann kann man es wohl auch durchsetzen, daß er sich im Interesse seiner Sicherheit, auch ohne operiert zu werden, in eine Krankenanstalt begibt.

Ich habe diesen Standpunkt schon gelegentlich verschiedener Diskussionen über die Behandlung der Appendicitis vertreten und halte ihn ebenso aufrecht wie meine gleichzeitig geäuberte Auffassung, daß bei Vorhandensein grober Abszesse diese auf dem kürzesten Wege, womöglich ohne Eröffnung der freien Bauchhöhle, entleert werden sollen, ohne die Appendix zu entfernen. Dazu veranlaßt mich einzig und allein die Absicht, den Eingriff möglichst ungefährlich zu gestalten, und nicht etwa die Auffassung, daß nach größeren Abszessen die Rezidive der Appendicitis selten seien. Meiner Erfahrung nach kommen auch nach großen Abszessen Rezidive oft genug vor, und ich komme bei Besprechung der Intervall-Operation noch darauf zurück. Daß man nach einfacher AbszeBinzision ab und zu noch sekundäre Abszesse (z. B. Douglasabszesse, subphrenische, linkseitige Abszesse) eröffnen müsse, ist ja richtig. Aber erstens ist das Vorkommnis selten, und zweitens schützt auch die Radikaloperation im intermediären Stadium davor nicht, ja selbst nach Frühoperation sind in ganz vereinzelten Fällen später noch Abszeßinzisionen erforderlich geworden.

Ich habe unter den 112 einfachen Inzisionen vier verloren und muß diese vier Todesfälle analysieren. Ein Mann starb 14 Tage nach der Inzision eines großen, zwei Wochen alten appendizitischen Abszesses an einer Embolie der Pulmonalarterie. Ein Patient, bei dem noben allgemein peritonitischen Erscheinungen ein Douglasabszeb deutlich nachweisbar war und bei dem ich nur den DouglasabszeB rektal inzidierte, starb an der Peritonitis. $\mathrm{Ob}$ ich den Fall durch Radikaloperation gerettet hätte, weiß ich nicht; jedenfalls hätte ich in diesem Falle, da neben dem Abszeb peritonitische Erscheinungen bestanden, laparotomieren sollen. Ich hielt aber damals - der Fall spielt vor mehreren Jahren - die Erscheinungen nur für Reizerscheinungen vom Abszeb aus und beging damit einen Fehler. Ich scheute mich aber, bei der deutlichen Nachweisbarkeit des Abszesses per rectum von oben einzugehen, weil zwei meiner Todesfälle nach Abszeßinzisionen eben durch die freie Bauchhöhle hindurch eröffnete Douglasabszesse betrafen. Sie starben beide an foudroyanter Peritonitis. Daß ich eine ganze Anzahl der gut abgelaufenen 108 Abszeßinzisionen durch die freie Bauchhöhle hindurch vornehmen mußte und bei entsprechender Abdämmung keinen weiteren Unglücksfall erlebte, hat mich doch nicht von der Auffassung abbringen können, daß die möglichst extraperitoneale Eröffnung des Abszesses ungefährlicher und daher vorzuziehen ist.

Ich möchte hier einige Bemerkungen über das Schicksal der nicht operierten appendizitischen Abszesse einschalten. Nur ein Teil derselben perforiert, wobei die Perforation in unglücklichen Fällen in die freie Bauchhöhle, in den glücklichsten Fällen an die freie Körperoberfläche oder in das Rectum, sonst in höhere Darmabschnitte, mitunter einmal in die Blase etc. erfolgen kann. Die von Sahli angenommene Selbstdrainage durch die Appendix kann vielleicht einmal - als allergrößte Seltenheit - vorkommen; wer die Verhältnisse durch die operative Autopsie genau kennt, wird die Eignung des destruierten Wurmfortsatzes als Drainagerohr nicht hoch bewerten. Eher kann bei Ablösung der Appendix vom Coecum das in diesem Darmabschnitt jetzt resultierende Loch dem Eiter als Abflußweg dienen. - Bleibt die Perforation des Abszesses aus, wird er nicht inzidiert, geht der Kranke nicht zugrunde, so erfolgt die Resorption des Eiters, und oft genug finden wir bei der nach Wochen oder Monaten ausgeführten Intervalloperation die krümligen Eiterreste in der Umgebung der Appendix. Wo unter allmählichem Nachlassen der entzündlichen Erscheinungen, langsamem Abfall der Temperatur, allmählichem Nachlassen der Schmerzhaftigkeit die fühlbare Resistenz von Tag zu Tag kleiner wird, liegt ja die Annahme einer allmählichen Resorption des Eiterherdes ebenso nahe, wio anderseits bei raschem, sozusagen über Nacht erfolgendem Schwinden aller objektiv nachweisbaren Symptome - Schmerz, Fieber, Tumor - die Annahme der Perforation in ein benachbartes Hohlorgan (Darm) zunächst liegt. Letztere Annahme triff aber meines Erachtens nicht immerzu. Es kommt zweifellos ein derartiges fast plötzliches Verschwinden des Exsudates, allerdings nur ein scheinbares Verschwinden, auch ohne Perforation des Eiters vor. Es verkleinert sich in derartigen Fällen der palpable Tumor innerhalb von 12-24 Stunden unter Verschwinden der akutentzündlichen Erscheinungen, der AbszeB erkaltet sozusagen über Nacht, eine Erscheinung, wie wir sie auch bei Abszessen an anderen Körperstellen mitunter beobachten können und auf die ich an anderer Stelle näher eingehen werde.

Betonen will ich, wie ich dies schon seinerzeit getan habe und womit Sprengels Auffassung übereinstimmt, daß nebst den Allgemeinerscheinungen seitens Herz und Sensorium der Zustand des Darmes für die Prognose entscheidend ist und daß hochgradiger Meteorismus, speziell bei älteren Leuten, die Prognose aufs äußerste trübt.

Merkwürdig divergente Anschauungen hört man noch immer über die Indikation zur Intervalloperation äußern. Meine Erfahrungen beziehen sich auf 1063 Operationen, der weitaus größere Teil derselben nach klinisch sicher gestellten schweren Anfällen, nach Abszeßinzisionen etc. ausgeführt, mit fünf Todesfällen. Von diesen Todesfällen betrafen drei Peritonitiden, die von gelegentlich der Operation zwischen schwieligen Verwachsungen aufgedeckten Eiterresten ausgegangen waren, einmal erfolgte der Tod an lleus (es wurden größere Strecken des Dünndarms des Serosaüberzuges beraubt), und ein Todesfall an Peritonitis (ohne Sektion) erfolgte nach einer Operation, die eine mit Kot prall gefüllte, hypertrophische, aber ganz freie, an der Serosafläche unveränderte Appendix beseitigt hatte. Die Gefahr der Operation ist also sehr gering, und das erleichtert den Entschluf zur Operation sehr, um so mehr, als ja relativ gröBere Gefahren nur dort entstehen, wo die Operation schwer war, eine schwer erkrankte Appendix ergeben hatte, wo also die Indikation keine relative war.

Es ist bemerkenswert, dal einzelne Chirurgen meinen, die Appendektomie à froid sei nach leichten Anfällen überflüssig, weil die Appendix wieder ganz normal werden könne, während eine größere Reihe von Chirurgen wieder die à froid-Operation nach sehr schweren, mit grofen Exsudaten verlaufenden Attacken für überflüssig erklärt, weil 1. nach solchen Attacken der Wurmfortsatz durch Verwachsungen seine Gefährlichkeit für spätere Zeiten eingebüßt habe und weil 2. in so schweren Attacken der Wurmfortsatz oft zugrunde gehe. All diese Behauptungen, von so autoritativer Seitesie auch aufgestellt werden, halten der klinischen Prüfung auf Grund meines Materials nicht stand. Was zunächst die sogenannten leichten, eventuell zur vollen Restitutio ad integrum führenden Attacken betrifft, so verbirgt sich hinter einer leichten Attacke oft genug ein UlzerationsprozeB um einen nahe der Mündung der Appendix gelegenen Kotstein. Der kurze Anfall ist mit der Ausstoßung des Kotsteines in das Coecum erledigt, aber aus dem Ulcus wird eine narbige Stenose, und dahinter entwickelt sich das Empyem. Oft genug habe ich nach Anfällen, die laut ärztlichem Ausspruch ganz leicht gewesen waren, à froid operiert und nun eine Ap- 
pendix entfernt, die eine ganz locker verklebte, eventuell nur durch adhärentes Netz gedeckte Perforation trug. Finden wir doch oft genug ohne jeden anamnestisch sicher gestellten Anfall tiefe Narben - kein überraschender Befund. wenn wir bedenken, daßauch an anderen Darmpartien Ulzerationsprozesse ganzsymptomlos verlaufen können. Ebensowenig darf man nach schweren Attackendio Intervalloperation ablehnen. Daf nach Abszessen Rezidive selten sind, kann ich nach meiner persönlichen Statistik nicht bestätigen. Ich bin ja natürlich durch die Intervalloperation vielen Rezidiven zuvorgekommen, aber ich verfüge über eine große Reihe von Fällen, in welchen nach Durchbruch oder Inzision eines Abszesses neuerlich Abszesse oder schwere Attacken mit Allgemeinerscheinungen auftraten. Und daf nach schweren Attacken ein vollkommenes Zugrundegehen der Appendix häufig sei, ist eine Fabel. Unter meinen zahlreichen Intervalloperationen, die großenteils nach sehr schweren Anfällen unternommen worden waren, habe ich ein einzigesmal keine Appendix mehr gefunden. In allen anderen Fällen fand ich die mehr oder weniger schwer veränderte, stets zur Erregung einer neuen Attacke hinreichend konservierte Appendix. Darunter befanden sich auch zwei Fälle, in denen während früherer Anfälle anderwärts ein Abszeb entleert und nach Angabe des betreffenden Chirurgen die Appendix mit dem Eiter ganz ausgestoben worden war! In acht Fällen war die Appendix komplett vom Coecum amputiert und mehr oder weniger weit von ihm dreimal im Coecum Douglasii - zu finden.

Also die Erfahrung zeigt, daß auch nach schweren und wiederholten Anfällen die Appendix fortexistiert, und weder ausgedehnte Exsudate, noch der Abgang von Kotsteinen mit dem Eiter schließt eine neuerliche Attacke von Appendicitis aus. Aber auch eine einfache Ueberlegung zeigt, wie falsch die Theorie von der vollständigen Zerstörung der Appendix im Verlauf einer schweren appendizitischen Attacke ist. Was genügt denn zu einem schweren Anfall? Doch jede zirkumskripte, tiefgehende Zerstörung, eine stecknadelkopfgroße Perforation der Appendix! Es bedarf doch keiner Zerstörung eines größeren Appendixabschnittes zur Erzeugung einer Peritonitis! Und nach Ausstoßung eines Kotsteines kann die Narbe - wie ja auch Sprengel erwähnt - irgendwo im zentralen Anteil der Appendix zurückbleiben, und der peripherische Teil bleibt gefahrdrohend zurück. Da aber auch ein schwerer Anfall nicht davor schützt, daß der nächste Anfall ebenso schwer ist, da mir z. B. drei Fälle bekannt sind, die nach glücklichem Verlauf eines resp. zweier schwerer Anfälle in einem dritten Anfall zugrunde gegangen sind, so entfällt jede Berechtigung, für Einschränkung der Intervalloperation nach schweren Anfällenzu plädieren. Jeder leichte, jeder schwere, jeder einmalige Anfall einer Appendicitis berechtigt zur Intervalloperation, die nach moiner Statistik viol ungefährlichor ist als die Frühoperation $(0,47 \%$ Mortalität gegen über $6,5 \%)$.

Nur sollte der entscheidende Anfall stets zweifellos diagnostiziert sein! Ich kann aber auch nicht zugeben, dab die Intervalloperationen nach vorhergegangenen schweren Attacken sehr kompliziert sind (Lenhartz), vielmehr ist es in der Regel überraschend, wie wenig Verwachsungen die abgelaufene Peritonitis zurückgelassen hat.

Auf alle Komplikationen: Gravidität, Erkrankungen der Adnexe - hier handelt es sich übrigens fast nie um einen ätiologischen Zusammenhang -, Ikterus, Haematemesis etc. kann ich hier nicht eingehen. Nur über den DarmverschluB im Verlauf der Appendicitis und nach Operation wegen Appendicitis muß ich ein paar Bemerkungen machen, und diese sollen sich in erster Linie auf die Therapie beziehen. Ich habe die Enterostomie beim peritonitischen DarmverschluB schon im Februar 1901 1) warm empfohlen. Wenige Monate später ist Heidenhain sehr energisch für dieses Verfahren eingetreten, und seither ist es vielfach nachgeprüf, teils anerkannt, teils als wertlos verurteilt worden.

Meine Erfahrungen beziehen sich bis zum Beginn dieses
Jahres auf 55 Enterostomien bei resp. nach Laparotomien wegen Appendicitis. darunter 4 doppelte. Von den 51 einfachen Enterostomien sind 32 geheilt. 19 gestorben. von den 4 mehrfachen Entrostomien sind 3 geheilt, 1 gestorben. Aus der groben Zahl und den guten Resultaten können Sie entnelımen. dab ich mit der Vornahme der Enterostomie nicht lange zögere. Fehlt der Abgang von Stuhl und Winden, bleiben Klismen erfolglos, tritt sichtbare Darmsteifung auf, oder lassen sich schwi ppende Darmschlingen nachweisen. so enterostomiere man. Die besten Resultate geben jene Fälle, die sich die ersten zwei bis drei Tage nach der Operation ganz wohl befinden und dann erst die allmählich slch steigernden Symptome des Darmverschlusses aufweisen. Man warte aber nicht, bis Dünndarminhalt erbrochen wird, sondern operiere bei den ersten unzweideutigen Symptomen. Und man begnüge sich mit der Enterostomie der geblähten Schlinge und vermeidejedes Herumsuchen nach Sitz und Art des Hindernisses. Wer spät und eingreifend operiert, wird schlechte Resultate haben, nur wer früh und schonend eingreift, wird beim postappendizitischen Darmverschlub gute Erfolge aufweisen. Auch auf diese Frage muß ich mir versagen hier genauer einzugehen, es soll dies an anderer Stelle geschehen.

Zu weit würde es auch führen, wollte ich an dieser Stelle auf die Technik der Appendektomie, auf die Hernienbildung. auf die Drainage etc. eingehen. Auch das soll anderwärts eingehend geschehen. An dieser Stelle wollte ich nur auf Grund meiner persönlichen Erfahrungen einen ungeschminkten Rechenschaftsbericht abgeben und über einzelne strittige Punkte meine Auffassung äußern. Hierzu ist meiner Ansicht nach, trotz der enormen Appendicitis-Literatur, jeder Chirurg verpflichtet, dem eine besonders große Zahl von Appendicitiskranken anvertraut war.

Zusammenfassung. Die Appendicitis, die übrigens nicht häufiger geworden 1st, entsteht in der Regel enterogen. Die hämatogene Entstehung ist eine seltene Ausnahme; auch nach Anginen etc. entstehende Appendicitiden sind zumeist $\mathrm{nicht}$ hämat ogenen Ursprunges. Den Kotsteinen kommt sehr große Bedeutung nicht nur für den Verlauf, sondern auch für die Entstehung der Appendicitis zu. - Die Diagnose der chronischanfallsfreien Appendicitis ist sehr unsicher. Abgesehen von den bekannten Verwechslungen muß darauf aufmerksam gemacht werden, daß bei latenter Tuberkulose - speziell der Lungen - Schmerz und Druckempfindlichkeit in der Ileozökalgegend nicht selten ist. Die operative Behandlung des akuten Anfalles ist in jedem Falle, der nur irgend ein schweres Symptom aufweist, innerhalb der ersten 48 Stunden zu emplehlen. Im weiteren Verlaufe muß individualisierend vorgegangen werden. Grobe Abszesse sind auf dem kürzesten Wege, Douglasabszesse in erster Linie per rectum zu inzidieren. Die à froid-Operation ist, wenn auch nur ein vorausgegangener Anfall sichergestellt ist, $\mathrm{zu} e \mathrm{mpfehlen}$, sie ist aber auch nach den schwersten Anfällen zu empfehlen, da die Annahme, daß in schweren Anfällen der Wurmfortsatz zugrunde geht resp. sich abstößt, falsch ist. 